\title{
Erratum to: Hepatitis E Virus Genotype 4 Sequences Detected in Sewage from Treatment Plants of China
}

\author{
Heng $\mathrm{Li}^{1} \cdot$ Wei $\mathrm{Li}^{1} \cdot$ Ruiping She ${ }^{1,3} \cdot$ Liang $\mathrm{Yu}^{2} \cdot$ Qiaoxing $\mathrm{Wu}^{1} \cdot$ Jingling Yang $^{1} \cdot$ \\ Fengjiao $\mathrm{Hu}^{1}$ - Majid Hussain Soomro ${ }^{1}$ Ruihan Shi ${ }^{1}$ - Wenzhuo Hao ${ }^{1}$. \\ Yue Zhao ${ }^{1} \cdot$ Jingjing Mao'
}

Published online: 7 March 2017

(C) Springer Science+Business Media New York 2017

\section{Erratum to: Food Environ Virol DOI 10.1007/s12560-016-9276-y}

The original version of this article unfortunately contained a mistake in Fig. 1. Position of G3 and G4 was reversed. The corrected Fig. 1 is given below.

The online version of the original article can be found under doi:10.1007/s12560-016-9276-y.

Ruiping She

sheruiping@126.com

Heng $\mathrm{Li}$

283578425@qq.com

Wei Li

1016424331@qq.com

Liang $\mathrm{Yu}$

2117970499@qq.com

Qiaoxing Wu

924813953@qq.com

Jingling Yang

1091565008@qq.com

Fengjiao Hu

775527474@qq.com

Majid Hussain Soomro

majidsoomro@hotmail.com

Ruihan Shi

845209132@qq.com
Wenzhuo Hao

1021869647@qq.com

Yue Zhao

15416350@qq.com

Jingjing Mao

315078314@qq.com

1 Laboratory of Veterinary Pathology and Public Health, College of Veterinary Medicine, China Agricultural University, Yuanmingyuan WestRoad 2\#, Haidian District, Beijing 100193, China

2 Shenzhen Urban Wastes Disposal \& Recycling Center, Yuyuan Road 1\#, Longgang District, Shenzhen 518000, China

3 Laboratory of Animal Pathology \& Public Health, Key Laboratory of Zoonosis of the Ministry of Agriculture College of Veterinary Medicine, China Agricultural University, Beijing 100193, China 
Fig. 1 Phylogenetic analysis based on ORF2 (348 nt) depicting the genetic relationship between our strain from this study. A neighborjoining tree was constructed with bootstrap values calculated from 1000 replicates. Isolates used for comparative analysis were Burma (GenBank Accession No. M73218, D10330), Pakistan (AF185822), India (X99441), India (X98292), Pakistan (M80581), China (D11092), Eygypt (AF051352), Mexico (M74506), China (GU119961), China (HQ634346), China (AJ272108), China (AY594199), Japan (AB481229), USA(AF060669), USA (AF060668), and Avian HEV (AY535004)

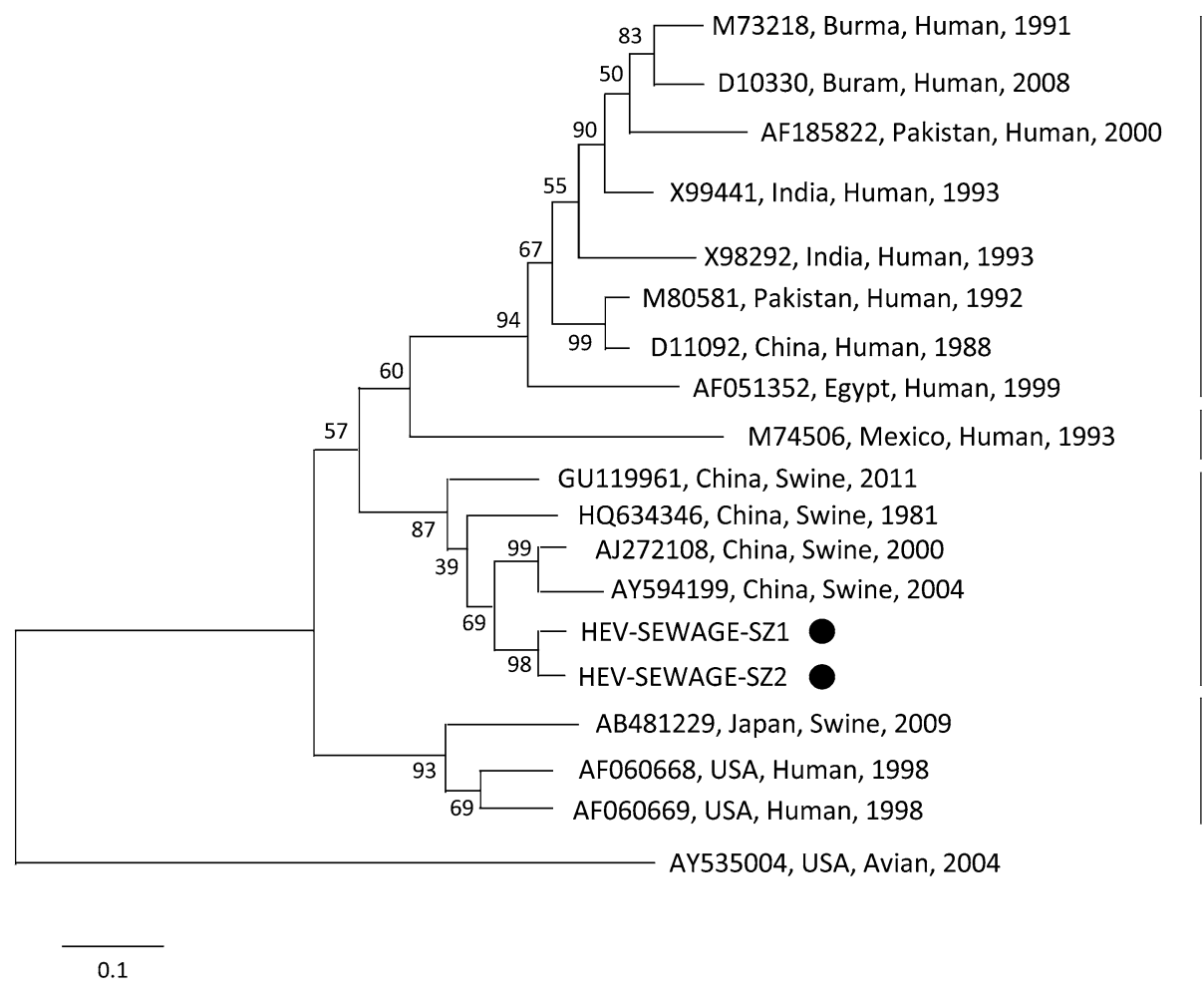

